\title{
A study on diffusion tensor imaging in patients with untreated first-episode obsessive-compulsive disorder
}

\author{
Bai-Ling Huang ${ }^{1}$, Jun-Ru Wang ${ }^{1}$, Xu-Huan Yang ${ }^{1}$, Yu-Ming Ren ${ }^{2}$, Hui-Rong Guo ${ }^{1}$ \\ ${ }^{1}$ Department of Psychiatry, The First Affiliated Hospital of Zhengzhou University, Zhengzhou, China; ${ }^{2}$ Office of Academic Studies, Xinxiang \\ Medical University, Xinxiang, China
}

Contributions: (I) Conception and design: BL Huang; (II) Administrative support: JR Wang; (III) Provision of study materials or patients: XH Yang; (IV) Collection and assembly of data: YM Ren; (V) Data analysis and interpretation: HR Guo; (VI) Manuscript writing: All authors; (VII) Final approval of manuscript: All authors.

Correspondence to: Hui-Rong Guo, MD. Department of Psychiatry, The First Affiliated Hospital of Zhengzhou University, Zhengzhou 450052, China. Email: ruirongguo123@163.com; Yu-Ming Ren, MD. Office of Academic Studies, Xinxiang Medical University, Xinxiang 453003, China. Email: rnyumimg@21cn.com.

Objective: The present study provides an overview of studies investigating white matter (WM) integrity in patients with obsessive-compulsive disorder (OCD) using diffusion tensor imaging (DTI). Furthermore, it studies the correlation of fractional anisotropy (FA) in abnormal cerebral WM areas with the course and clinical signs of the disease.

Methods: The study subjects were divided into two groups, the OCD group $(n=38)$ and the control group $(\mathrm{n}=40)$, based on the Diagnostic and Statistical Manual of Mental Disorders 5 (DSM-5) diagnostic criteria for OCD. Patients with untreated first-episode OCD were assigned to the OCD group, while healthy volunteers were assigned to the control group. The study group was evaluated in accordance with the YaleBrown Obsessive-Compulsive Scale (Y-BOCS), Self-Rating Depression Scale (SDS) and Self-Rating Anxiety Scale (SAS). Subjects who met the inclusion criteria underwent whole-brain scanning via $3.0 \mathrm{~T}$ structural magnetic resonance imaging (sMRI). The WM FA values in different brain areas were compared between the two groups using voxel-based analysis (VBA). Subsequently, the correlations of the patient Y-BOCS score and disorder course with the FA values in significantly improved encephalic areas were analyzed.

Results: (I) The FA values of the right precentral gyrus (PreCG.R), left insular lobe, left inferior frontal gyrus and right inferior occipital gyrus (Occipital_Inf_R) WM were significantly lower in the OCD group than in the control group $(\mathrm{P}<0.05)$. Elevated FA values were not observed in the OCD group. (II) FA values of PreCG.R, left insular lobe/left inferior frontal gyrus, and Occipital_Inf_R were not found in relation to the total $\mathrm{Y}-\mathrm{BOCS}$ score $(\mathrm{P}=0.122 ; \mathrm{P}=0.401 ; \mathrm{P}=0.134)$, obsessional thoughts score $(\mathrm{P}=0.299 ; \mathrm{P}=0.760$; $\mathrm{P}=0.062)$, compulsive activities checklist $(\mathrm{P}=0.487 ; \mathrm{P}=0.420 ; \mathrm{P}=0.431)$, and disease course $(\mathrm{P}=0.604 ; \mathrm{P}=0.380$; $\mathrm{P}=0.182)$.

Conclusions: Multiple microstructural cerebral WM changes were observed in the frontal lobe, occipital lobe, and insula in patients with untreated first-episode OCD, presenting the correlation of these changes with OCD occurrence.

Keywords: Obsessive-compulsive disorder (OCD); white matter (WM); magnetic resonance imaging (MRI); diffusion tensor imaging (DTI)

Submitted Jun 29, 2021. Accepted for publication Sep 13, 2021.

doi: 10.21037/qims-21-682

View this article at: https://dx.doi.org/10.21037/qims-21-682 


\section{Introduction}

Obsessive-compulsive disorder (OCD) is a common disabling chronic psychiatric disorder, with recurrent intrusive obsessive thoughts and uncontrollable compulsive behaviors as the main clinical manifestations (1). The general population incidence is approximately $2-3 \%$ (2). The disorder is characterized by a high disability rate, low cure rate and treatability, severely impaired social functioning, and complex clinical manifestations. According to the World Health Organization, OCD is the tenth most disabling disease to live with. Presently, the etiology and pathogenesis of the disorder remain unspecified. Early identification of OCD is mainly through clinical manifestations and psychological evaluation. Interventions include medication, psychotherapy and physical therapy. Selective serotonin reuptake inhibitors (SSRIs) and cognitive behavioral therapy (CBT) are currently recognized as first-line treatments for OCD.

Diffusion tensor imaging (DTI) is a noninvasive imaging technique that clearly shows the running of cerebral white matter (WM) fiber tracts in the deep brain tissue by indirectly assessing the structural integrity of the cerebral WM through the evaluation of water molecule diffusion. It is also an important method for studying cerebral WM structure changes. The commonly used parameters include fractional anisotropy (FA), mean diffusivity (MD), radial diffusivity (RD) and axial diffusivity (AD), the FA is the most sensitive and commonly used parameter to describe the changes of tissue microstructure (3). FA is the most sensitive and commonly used parameter for describing microstructural changes in brain tissue (4). Other parameters that can be used to describe DTI include $\mathrm{MD}, \mathrm{AD}$ and $\mathrm{RD}$; because they are not as convenient and clear as FA images in image generation, they are less used in clinical research.

Many studies at home and abroad on DTI in patients with OCD have reported changes in cerebral WM; however, different studies have found inconsistent FA values, which were either elevated (5), decreased (6), or normal (7), and there are also differences in the involved abnormal brain regions. It remains unclear whether abnormal cerebral WM exists in other brain areas than those in which it is known so far. Further exploration is necessary in order to provide an imaging basis for the etiological study of OCD, along with early identification and intervention of the disease. We present the following article in accordance with the MDAR reporting checklist (available at https://dx.doi.org/10.21037/ qims-21-682).

\section{Methods}

\section{Materials}

\section{OCD group}

Outpatients and inpatients with first-episode OCD, none of whom were ever treated in any way, who visited the Psychiatry Department of the First Affiliated Hospital of Zhengzhou University between November 2018 and November 2020 were enrolled in the present study.

Inclusion criteria: (I) patients who met the Diagnostic and Statistical Manual of Mental Disorders 5 (DSM-5) diagnostic criteria for OCD, and does not meet the diagnostic criteria of other mental disorders such as depression, anxiety disorder or schizophrenia; (II) patients with a Yale-Brown Obsessive-Compulsive Scale (Y-BOCS) score of $\geq 16$, Self-Rating Depression Scale (SDS) score of $<53$, and Self-Rating Anxiety Scale (SAS) score of $<50$; (III) patients who were presently experiencing their first episode and who had not been previously treated in any way; (IV) patients of Han ethnicity; (V) patients who were right-handed; and (VI) patients with a primary or higher education level. Age and sex were not restricted.

A total of 56 patients with OCD were initially received for the study. Of these, 14 did not undergo a magnetic resonance imaging (MRI), and 4 were excluded because of magnetic susceptibility artifacts due to tooth wear, image registration deformation, and wide-flow-related artifacts. Finally, 38 patients were included in the OCD group for analysis.

\section{Control group}

Healthy volunteers were recruited into the control group through advertisement during the same phase as the patients in the OCD group.

Inclusion criteria: (I) subjects without OCD and other psychotic disorders, determined in accordance with the DSM-5 diagnostic criteria; (II) subjects without a family history of psychotic disorders; (III) subjects of Han ethnicity; (IV) subjects who were right-handed; and (V) subjects with a primary or higher education level. Age and sex were not restricted. A total of 40 subjects were included in the control group.

\section{Common exclusion criteria}

Exclusion criteria for both groups: (I) subjects with mental retardation; (II) subjects with previous or current psychiatric disorders, nervous system disorders, or severe physical diseases; (III) subjects with previous or current alcohol 
or drug dependency; (IV) subjects with MRI examination contraindications; (V) subjects with routine MRI imaging showing brain structure abnormalities; (VI) subjects who had primary relatives with histories of psychiatric disorders; and (VII) pregnant and lactating women.

The study was conducted in accordance with the Declaration of Helsinki (as revised in 2013). The study was approved by the ethical committee of the First Affiliated Hospital of Zhengzhou University and informed consent was taken from all the patients.

\section{Diagnosing and evaluating using rating scales}

The diagnoses of the enrolled subjects were made by one associate chief physician and above from the psychiatry department in accordance with the DSM-5 diagnostic criteria for OCD. Two psychiatry department physicians who received training for rating scale consistency evaluated patients with OCD using Y-BOCS and provided guidance for completing the evaluation using SDS and SAS.

\section{MRI data acquisition}

The routine T1-weighted imaging and DTI scanning for all subjects were conducted by one well-experienced and welltrained MRI physician. The scanning was completed on the same MRI scanner using the GE Discovery $7503.0 \mathrm{~T}$ MRI imaging system (GE Healthcare, Anaheim, CA, USA) in MRI room\#1 of the First Affiliated Hospital of Zhengzhou University. Before the scanning, the physician informed all subjects about what to do and what not to do during the scanning in order to ensure subject cooperation and, thus, examination completion. Subjects were placed in the supine position and provided with earmuffs to block out scanner noise and a foam pad to minimize head motion during the scanning. Subjects were instructed to stay still, close their eyes, keep awake, and try not to think about anything. The routine scanning parameters were as follows: repetition time $(\mathrm{TR})=8.2 \mathrm{~ms}$; echo time $(\mathrm{TE})=3.2 \mathrm{~ms}$; flip angle $=12^{\circ}$; matrix $=256 \times 256$; field of view $(\mathrm{FOV})=256 \mathrm{~mm} \times 256 \mathrm{~mm}$; slice thickness $=1.0 \mathrm{~mm}$; slice interval $=0 \mathrm{~mm}$; and layer number $=188$.

Parameters for DTI scanning were as follows: $\mathrm{TR}=7,100 \mathrm{~ms}$; inversion time $(\mathrm{TI})=2,025 \mathrm{~ms}$; $\mathrm{TE}=61 \mathrm{~ms} ; \mathrm{FOV}=256 \mathrm{~mm} \times 256 \mathrm{~mm}$; slice thickness $=2 \mathrm{~mm}$; slice interval $=0 \mathrm{~mm}$; layer number $=70$; matrix $=128 \times 128$; acquisition time $=8 \min 53 \mathrm{~s} ; \mathrm{FA}=90 ; \mathrm{B}$ values $=1,000 \mathrm{~s} / \mathrm{mm}^{2} ;$ and diffusion gradient directions $=64$.

\section{MRI data processing}

All data were processed based on Linux and using Statistical Parametric Mapping (SPM8) and software tools from the Functional MRI of the Brain (FMRIB) Software Library (FSL, version 6.0). The specific processing procedures were as follows: (I) the original MRI DICOM images were transferred into the NIFTI format using dcm2nii. (II) The eddy current FSL correction toolbox was applied in order to pre-align the diffusion dataset and correct the head motion effect and eddy current. (III) The FSL diffusion toolbox (FDT) was utilized to calculate the diffusion tensor of every voxel. (IV) Individual parameters registered into standard space: the obtained FA image was registered into MNI standard space using SPM8. (V) The re-sampling of all images with diffusion parameters was performed with a spatial resolution of $2 \mathrm{~mm} \times 2 \mathrm{~mm} \times 2 \mathrm{~mm}$ voxel size. (VI) Image smoothing standardization: a $6 \mathrm{~mm}$ halfwidth Gaussian was used to verify all transformed FA images for the smoothing process in order to reduce the space noise and compensate for standardization-induced inaccuracies. The multiple comparison correction for statistical results was conducted using Gaussian random field (GRF) theory; there existed significant differences $(\mathrm{P}<0.05)$. Next, a voxelbased statistical analysis was performed for the obtained DTI parameter maps using SPM8. Here, differences in the obtained data were displayed in graphs using the xjview software.

\section{Statistical analysis}

The general data and clinical data statistical analyses were performed using the Statistical Package for Social Sciences 22.0 (IBM Corp., Armonk, NY, USA). In the comparisons between the two groups, the Mann-Whitney $\mathrm{U}$ test was used for age, the two independent samples $t$-tests were used for education years, and the $\chi^{2}$ test and Chi-square test were used for gender. The normal distribution measurement data were expressed as mean \pm standard deviation $(\bar{x} \pm S)$ and the measurement data of non-normal distribution were expressed as median and quartiles (M [P25, P75]). The correlation analyses of the FA values of abnormal brain areas with significant differences as well as the compulsive activities checklist, disease course, total Y-BCOS scores, and obsessional thoughts scores in the two groups were analyzed. The Pearson correlation analysis was applied if the normal distribution was met; otherwise, the Spearman correlation analysis was utilized. The differences were statistically significant $(\mathrm{P}<0.05)$. 
Table 1 Demographic data and clinical features in OCD group and control group

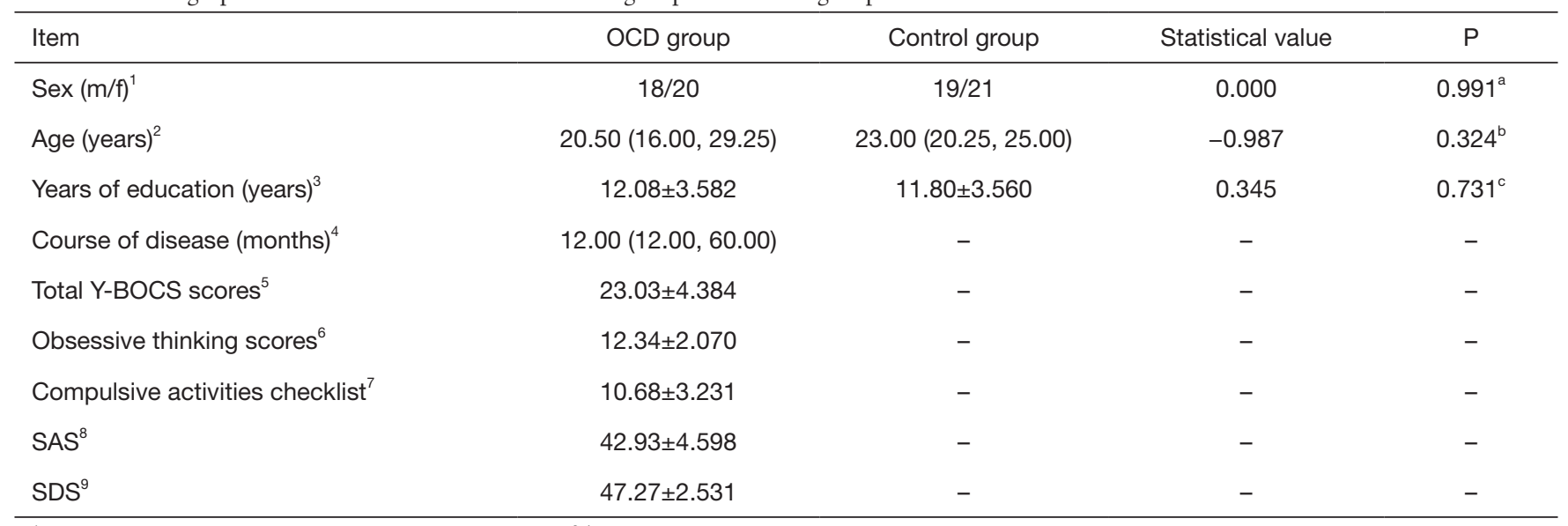

${ }^{1}$, the results were expressed as (male/female); ${ }^{2,4}$, the results were expressed as median (inter quartile range $P_{25}$, inter quartile range $\left.\mathrm{P}_{75}\right) ;{ }^{3,5-9}$, the results were expressed as (mean \pm standard deviation); ${ }^{a}, \chi^{2}$ test; ${ }^{b}$, Mann-Whitney $\cup$ test; ${ }^{\circ}, t$-test; - , not applicable. OCD, obsessive-compulsive disorder; Y-BOCS, Yale-Brown Obsessive-Compulsive Scale; SAS, Self-Rating Anxiety Scale; SDS, Self-Rating Depression Scale.

\section{Results}

\section{Demographic data and clinical features in the two groups}

The present study included a total of 78 subjects, 38 of whom had OCD and 40 of whom were healthy control subjects. There were no statistically significant differences between the two groups regarding gender $\left(\chi^{2}=0.000\right.$; $\mathrm{P}=0.991)$, age $(\mathrm{Z}=-0.987 ; \mathrm{P}=0.324)$, and education year number $(t=0.345 ; \mathrm{P}=0.731)$ (Table 1$)$.

\section{FA value comparison between the two groups}

Voxel-based analysis (VBA) was used to statistically analyze the brain regions with differences between the two groups. The FA values of WM in the PreCG.R, left insular lobe, left inferior frontal gyrus, and Occipital_Inf_R were significantly reduced in the OCD group compared with the control group; the differences were statistically significant $(\mathrm{P}<0.05)$. Elevated FA values were not observed in the OCD group (Figure 1, Table 2).

\section{Correlation analysis}

No correlation was observed between the FA values of the above-listed abnormal brain areas and the total Y-BOCS score, obsessional thoughts score, compulsive activities checklist, and the disease course in all patients with OCD ( $\mathrm{P}>0.05)$ (Table 3).

\section{Discussion}

In the present study, DTI and the VBA were used to investigate cerebral WM microarchitecture abnormalities in patients with untreated first-episode OCD. The results showed that the FA values of PreCG.R, left insular lobe, left inferior frontal gyrus, and Occipital_Inf_R WM were significantly lower in the OCD group than in the control group, presenting extensive destruction of WM integrity in brain areas in patients with OCD. This includes not only the brain areas related to the classic cortico-striatalthalamic-cortical (CSTC) loop model but also the occipital lobe and insula in the brain areas beyond the classic loop. This indicates that WM microstructure abnormality is closely related to OCD occurrence and development.

The precentral gyrus is mainly responsible for processing the sensory input and motor output. This area may also influence the patient's lack of control over his/her behavior and further cause OCD signs and symptoms. A large number of structural and functional imaging studies at home and abroad have found that patients with OCD have abnormalities in the precentral gyrus area. The present study found that the FA value of PreCG.R WM was significantly lower in the OCD group than in the control group, indicating that there existed significant changes in PreCG.R WM microstructure in patients with OCD. Koprivová et al. (8) found a significant reduction in precentral gyrus gray matter volume in patients with OCD. 

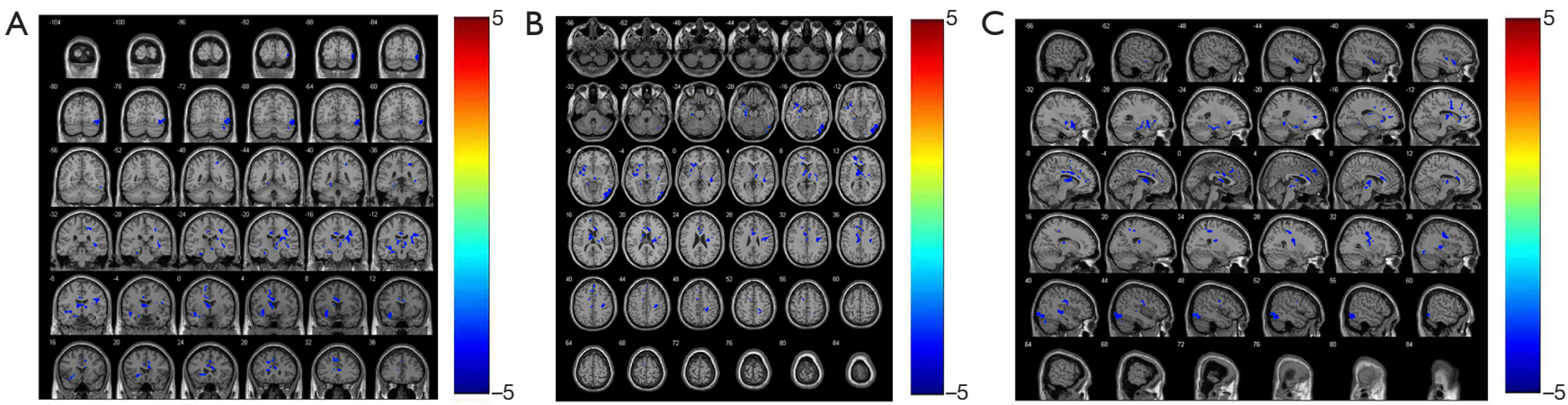

Figure 1 Brain area with FA difference among subject in two groups (A) coronal view; (B) axial view; (c) sagittal view. Red means elevated brain areas; blue means decreased brain areas. FA, fractional anisotropy.

Table 2 Brain areas with difference in OCD group and control group

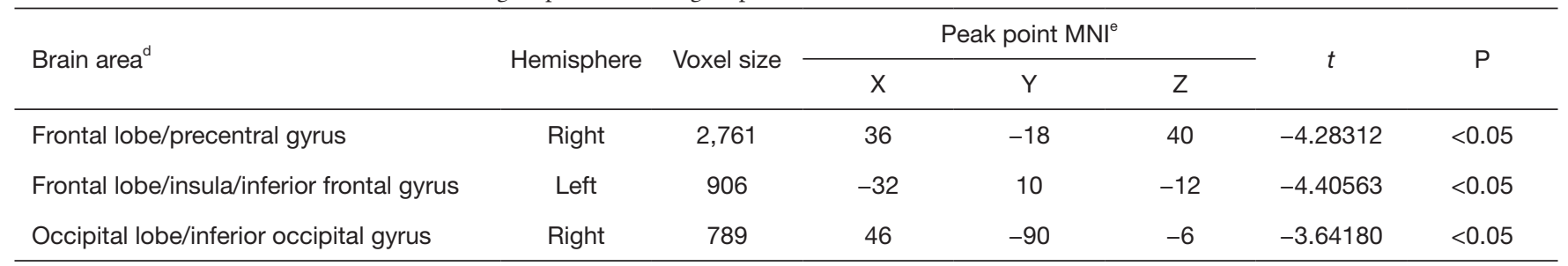

${ }^{d}$, the brain area with statistical difference, corrected by GRF, $\mathrm{P}<0.05$; ${ }^{\mathrm{e}}$, the coordinate of the most significant difference point. OCD, obsessive-compulsive disorder; GRF, Gaussian random field.

Table 3 Correlation between brain areas with difference and clinic features in OCD group

\begin{tabular}{|c|c|c|c|c|}
\hline Brain area & Total Y-BOCS scores ${ }^{\dagger}$ & Obsessive thinking scores ${ }^{g}$ & Compulsive activities checklist ${ }^{\dagger}$ & Course of disease ${ }^{g}$ \\
\hline$r / r_{s}$ & -0.255 & -0.200 & -0.116 & -0.087 \\
\hline $\mathrm{P}$ & 0.122 & 0.299 & 0.487 & 0.604 \\
\hline \multicolumn{5}{|c|}{ Left insular lobe/left inferior frontal gyrus } \\
\hline$P$ & 0.401 & 0.760 & 0.420 & 0.380 \\
\hline \multicolumn{5}{|c|}{ Occipital_Inf_R } \\
\hline$r / r_{s}$ & -0.248 & -0.305 & -0.132 & -0.221 \\
\hline $\mathrm{P}$ & 0.134 & 0.062 & 0.431 & 0.182 \\
\hline
\end{tabular}

${ }^{\mathrm{f}}$, Pearson correlation analysis; ${ }^{9}$, Spearman correlation analysis; $\mathrm{P}<0.05$. OCD, obsessive-compulsive disorder; Y-BOCS, Yale-Brown Obsessive-Compulsive Scale.

Mataix-Cols et al. (9) found that the precentral gyrus' activation function during symptom-induced tasks was significantly higher in patients with OCD than in healthy control subjects. The resting-state functional magnetic resonance imaging (rs-fMRI) study (10) found that the regional homogeneity ( $\mathrm{ReHo}$ ) of the precentral gyrus was significantly lower in patients with OCD than in healthy control subjects, indicating that neuron dysfunction in the precentral gyrus may play an important role in the pathological OCD mechanism. 
The present study found that the FA value of left inferior frontal gyrus WM was significantly reduced in the OCD group compared with the control group; this is consistent with the results of the study conducted by Yagi et al. (11), suggesting that WM abnormality in the left inferior frontal gyrus plays an important role in OCD pathogenesis. Similarly, Gonçalves et al. (12) found that the bilateral inferior frontal gyrus FA values were significantly lower in patients with OCD than in control subjects, while the $\mathrm{MD}$ and $\mathrm{RD}$ values were elevated, signifying that the inferior frontal gyrus is the core area involved in CSTC loop inhibitory control. The abnormalities might be related to the occurrence of obsessive-compulsive symptoms. Through the application of the cortical thickness analysis method, several scholars (13) found that the left inferior frontal gyrus cortex was thinner in patients with OCD than in control subjects. The inferior frontal gyrus is located in the ventral system and is mainly responsible for stimulation determination, meaning distribution, and behavior and motivation processing. Therefore, abnormalities in this area may result in the overestimation of stimulation, further leading to the occurrence of OCD, signified by meditation, excessive guilt, and persistent suspicion. In addition, in their research, Chen et al. (10) found that the rs-fMRI of patients with OCD showed a significant increase of ReHo in the left inferior frontal gyrus, suggesting that patients had a good coordination capability of neuronal activities in this area, which might be related to the constant recurrence of the obsessive-compulsive symptoms.

The present study further found that the FA value of the left insular lobe WM was significantly lower in the OCD group than in the control group. Functionally, the insula was connected with the dorsal anterior cingulate cortex, forming a salience network (SN). As a part of the $\mathrm{SN}$, the insular lobe was involved in the formation of receptive consciousness and the processing of movement and emotion, all of which were related to the occurrence of OCD symptoms. Zhu et al. (14) found that the insular lobe FA value in patients with OCD, characterized by symptom dimensions such as contamination/washing, was significantly lower than in the control subjects; this is consistent with the results of the present study, which further verifies the involvement of the abnormal insular WM in OCD pathogenesis. Certain studies (15) found that activation of the bilateral insular lobe, right superior frontal gyrus, and bilateral cerebellum was significantly better in patients with OCD compared with the control subjects; this promotes the theory that, in addition to the orbit frontal gyrus-striatum- thalamic loop, spontaneous neuronal activity abnormalities in the insular lobe and other brain areas may be the pathological basis of OCD. A structural MRI study (16) found that the insular lobe area gray matter volume in patients with OCD was significantly reduced. The correlation analysis found a relationship between insular lobe changes and the severity of OCD symptoms. These findings indicate that the uncontrollable obsessive thinking and compulsive behaviors in patients with OCD may be closely connected with brain monitoring dysfunction caused by abnormal insular area structure and function.

Furthermore, the present study found that the FA value of the Occipital_Inf_R WM was significantly lower in patients with OCD than in the control subjects. The occipital lobe is the key brain area for processing visual information and abstract concepts; its related brain areas are also correlated with cognitive function regulation. Damage to the occipital lobe and its related brain areas causes not only visual disorders but also memory defects and perceptual-motor disorders. Studies by Fan et al. (7) showed that the FA value of occipital lobe WM was significantly lower in patients with OCD than the control subjects. Ping et al. (15) found that the right inferior occipital gyrus ReHo was significantly reduced in patients with OCD compared with the study's healthy control subjects, indicating that spontaneous neuronal activity abnormality in the Occipital_Inf_R may be related to the emotional and behavioral dissonance of OCD. A study (17) on functional MRI found that the amplitude of lowfrequency fluctuations in the occipital lobe in patients with OCD was elevated in the resting state. Certain scholars (18) found that voxel-mirrored homotopic connectivity in the inferior occipital gyrus, lingual gyrus, and other brain areas was lower in patients with OCD than in the healthy control subjects, indicating that dysfunction in these brain areas may affect information transmission; this contributes to further understanding the neurobiological OCD occurrence mechanism. At present, there are relatively few reports on the occipital lobe and its related brain areas, with differences in results.

The present study observed no correlation between the FA value in abnormal cerebral WM areas in patients with OCD and the Y-BOCS scale score, obsessive thinking score, compulsive activities checklist, and disease course. This is inconsistent with the results of several previous studies (19) that found correlations between these factors. This may be due to the sample heterogeneity, different sample size, and analysis methods used. Further investigation with increased 
sample size and control over the impact of confounding factors is required in the future.

\section{Conclusions}

Changes in WM fiber tracts were observed in the frontal lobe, occipital lobe, and insula in patients with untreated first-episode OCD, presenting a close correlation of the changes with the occurrence of OCD. This study further demonstrated that changes in the related CSTC circuit brain areas may play an important role in the pathophysiological OCD mechanism and suggested that changes in wide brain areas, such as the occipital lobe and insula, are jointly involved in OCD pathogenesis.

\section{Limitations and outlook}

The present study has several limitations. Firstly, the FA values in patients with different symptom dimensions were compared neither by group nor longitude before and after treatment due to sample size limitations. Therefore, a larger sample size is required for future study. Secondly, the analysis method used in this study is relatively simple, and only changes in certain independent brain areas were observed. In the future, the use of MRI imaging technology that is more multimodal than the technology used in the present study is required in order to explain OCD pathogenesis from a more comprehensive perspective.

\section{Acknowledgments}

We would like to acknowledge the language editing of Melanie Tankard.

Funding: This study was supported by Science and Technology Department of Henan Province [172102310578]. The funding body had no role in the design of the study and collection, analysis and interpretation of data and in writing the manuscript.

\section{Footnote}

Reporting Checklist: The authors have completed the MDAR checklist. Available at https://dx.doi.org/10.21037/ qims-21-682

Conflicts of Interest: All authors have completed the ICMJE uniform disclosure form (available at https://dx.doi. org/10.21037/qims-21-682). The authors have no conflicts of interest to declare.

Ethical Statement: The authors are accountable for all aspects of the work in ensuring that questions related to the accuracy or integrity of any part of the work are appropriately investigated and resolved. The study was conducted in accordance with the Declaration of Helsinki (as revised in 2013). The study was approved by the ethical committee of the First Affiliated Hospital of Zhengzhou University and informed consent was taken from all the patients.

Open Access Statement: This is an Open Access article distributed in accordance with the Creative Commons Attribution-NonCommercial-NoDerivs 4.0 International License (CC BY-NC-ND 4.0), which permits the noncommercial replication and distribution of the article with the strict proviso that no changes or edits are made and the original work is properly cited (including links to both the formal publication through the relevant DOI and the license). See: https://creativecommons.org/licenses/by-nc-nd/4.0/.

\section{References}

1. de Salles Andrade JB, Ferreira FM, Suo C, Yücel M, Frydman I, Monteiro M, Vigne P, Fontenelle LF, TovarMoll F. An MRI Study of the Metabolic and Structural Abnormalities in Obsessive-Compulsive Disorder. Front Hum Neurosci 2019;13:186.

2. Chabardes S, Krack P, Piallat B, Bougerol T, Seigneuret E, Yelnik J, Fernandez Vidal S, David O, Mallet L, Benabid AL, Polosan M. Deep brain stimulation of the subthalamic nucleus in obsessive-compulsives disorders: long-term follow-up of an open, prospective, observational cohort. J Neurol Neurosurg Psychiatry 2020;91:1349-56.

3. Giraudo C, Cavaliere A, Lupi A, Guglielmi G, Quaia E. Established paths and new avenues: a review of the main radiological techniques for investigating sarcopenia. Quant Imaging Med Surg 2020;10:1602-13.

4. Gu BM, Park JY, Kang DH, Lee SJ, Yoo SY, Jo HJ, Choi CH, Lee JM, Kwon JS. Neural correlates of cognitive inflexibility during task-switching in obsessive-compulsive disorder. Brain 2008;131:155-64.

5. Li F, Huang X, Yang Y, Li B, Wu Q, Zhang T, Lui S, Kemp GJ, Gong Q. Microstructural brain abnormalities in patients with obsessive-compulsive disorder: diffusion-tensor MR imaging study at 3.0 T. Radiology 2011;260:216-23. 
6. He X, Steinberg E, Stefan M, Fontaine M, Simpson HB, Marsh R. Altered frontal interhemispheric and frontolimbic structural connectivity in unmedicated adults with obsessive-compulsive disorder. Hum Brain Mapp 2018;39:803-10.

7. Fan Q, Yan X, Wang J, Chen Y, Wang X, Li C, Tan L, You C, Zhang T, Zuo S, Xu D, Chen K, Finlayson-Burden JM, Xiao Z. Abnormalities of white matter microstructure in unmedicated obsessive-compulsive disorder and changes after medication. PLoS One 2012;7:e35889.

8. Koprivová J, Horácek J, Tintera J, Prasko J, Raszka M, Ibrahim I, Höschl C. Medial frontal and dorsal cortical morphometric abnormalities are related to obsessivecompulsive disorder. Neurosci Lett 2009;464:62-6.

9. Mataix-Cols D, Wooderson S, Lawrence N, Brammer MJ, Speckens A, Phillips ML. Distinct neural correlates of washing, checking, and hoarding symptom dimensions in obsessive-compulsive disorder. Arch Gen Psychiatry 2004;61:564-76.

10. Chen Y, Juhás M, Greenshaw AJ, Hu Q, Meng X, Cui H, Ding Y, Kang L, Zhang Y, Wang Y, Cui G, Li P. Abnormal resting-state functional connectivity of the left caudate nucleus in obsessive-compulsive disorder. Neurosci Lett 2016;623:57-62.

11. Yagi M, Hirano Y, Nakazato M, Nemoto K, Ishikawa K, Sutoh C, Miyata H, Matsumoto J, Matsumoto K, Masuda Y, Obata T, Iyo M, Shimizu E, Nakagawa A. Relationship between symptom dimensions and white matter alterations in obsessive-compulsive disorder. Acta Neuropsychiatr 2017;29:153-63.

12. Gonçalves ÓF, Sousa S, Maia L, Carvalho S, Leite J, Ganho A, Fernandes-Gonçalves A, Frank B, Pocinho F, Carracedo A, Sampaio A. Inferior frontal gyrus white

Cite this article as: Huang BL, Wang JR, Yang XH, Ren YM, Guo HR. A study on diffusion tensor imaging in patients with untreated first-episode obsessive-compulsive disorder. Quant Imaging Med Surg 2022;12(2):1467-1474. doi: 10.21037/qims21-682 matter abnormalities in obsessive-compulsive disorder. Neuroreport 2015;26:495-500.

13. Shin YW, Yoo SY, Lee JK, Ha TH, Lee KJ, Lee JM, Kim IY, Kim SI, Kwon JS. Cortical thinning in obsessive compulsive disorder. Hum Brain Mapp 2007;28:1128-35.

14. Zhu J, Li J, Han Y, Liu W. Relationship between symptom dimensions and white matter alterations in untreated patients with obsessive-compulsive disorder: a diffusion tensor imaging study. Neuroreport 2020;31:891-6.

15. Ping L, Su-Fang L, Hai-Ying H, Zhang-Ye D, Jia L, Zhi-Hua G, Hong-Fang X, Yu-Feng Z, Zhan-Jiang L. Abnormal Spontaneous Neural Activity in ObsessiveCompulsive Disorder: A Resting-State Functional Magnetic Resonance Imaging Study. PLoS One 2013;8:e67262.

16. Yoo SY, Roh MS, Choi JS, Kang DH, Ha TH, Lee JM, Kim IY, Kim SI, Kwon JS. Voxel-based morphometry study of gray matter abnormalities in obsessive-compulsive disorder. J Korean Med Sci 2008;23:24-30.

17. Zhu Y, Fan Q, Zhang H, Qiu J, Tan L, Xiao Z, Tong S, Chen J, Li Y. Altered intrinsic insular activity predicts symptom severity in unmedicated obsessive-compulsive disorder patients: a resting state functional magnetic resonance imaging study. BMC Psychiatry 2016;16:104.

18. Deng K, Qi T, Xu J, Jiang L, Zhang F, Dai N, Cheng Y, $\mathrm{Xu}$ X. Reduced Interhemispheric Functional Connectivity in Obsessive-Compulsive Disorder Patients. Front Psychiatry 2019;10:418.

19. Zhong Z, Yang X, Cao R, Li P, Li Z, Lv L, Zhang D. Abnormalities of white matter microstructure in unmedicated patients with obsessive-compulsive disorder: Changes after cognitive behavioral therapy. Brain Behav 2019;9:e01201. 